\title{
Seroprevalencia de Leptospira spp. en ganado bovino lechero de la región Huetar Norte de Costa Rica
}

\author{
Seroprevalence of Leptospira spp. in dairy cattle from the Huetar Norte Region in Costa Rica
}

\section{Jorge Sequeira Soto ${ }^{\square}$, María de los Ángeles Valverde Jiménez ${ }^{\mathrm{b}}$, Luis Nazario Araya} Sánchez ${ }^{c}$, José Alfredo Sequeira Avalos ${ }^{d}$, Manrique Oviedoe

a Centro Nacional de Referencia de Leptospirosis, Instituto Costarricense de Investigación y Enseñanza en Nutrición y Salud, INCIENSA. jsequeira@inciensa.sa.cr

b Centro Nacional de Referencia de Leptospirosis, Instituto Costarricense de Investigación y Enseñanza en Nutrición y Salud, INCIENSA. mvalverde@inciensa.sa.cr

c Escuela de Medicina Veterinaria. Universidad Nacional. Costa Rica. luis.araya.sanchez@una.cr

- Programa Salud de Hato, Cooperativa de Productores de Leche Dos Pinos R.L. asequeira@dospinos.com

e Programa Salud de Hato, Cooperativa de Productores de Leche Dos Pinos R.L. moviedo@dospinos.com

Recibido: 9 de Octubre de 2015. Corregido: 28 de Marzo de 2016. Aceptado: 6 de Mayo de 2016.

Resumen: Se realizó un estudio transversal, en la región Huetar Norte de Costa Rica, para determinar la seropositividad a Leptospira spp en ganado bovino lechero (2010-2012). Esta región presenta alta incidencia de la infección en humanos. Es la de mayor producción lechera en el país. Se procesó 2375 muestras de suero por la técnica del microaglutinación (MAT), correspondientes a 36 fincas lecheras asociadas a la Cooperativa de Productores de Leche Dos Pinos. La seroprevalencia global detectada, al considerar como positivos aquellos títulos mayores a 1:160, fue de $9.13 \%$, observándose títulos de hasta 1:5120 en animales clínicamente sanos. El comportamiento, entre fincas, fue heterogéneo, detectándose porcentajes de positividad entre $0-25 \%$ en los animales analizados. Los serogrupos, más frecuentemente identificados, correspondieron a Pomona (15.7\%), Tarassovi (16.6\%) y Hebdomadis (25.8\%). Se detectó una asociación, estadísticamente significativa $(p<0.05)$ con serogrupos asociados a algunas de las fincas muestreadas. De igual manera, las fuentes de agua utilizadas por las fincas presentaron asociación estadística $(p<0.05)$ con la positividad hacia Leptospira spp. Esta asociación no se identificó para la edad o la raza del animal ( $p>0.05)$.

Palabras clave: leptospirosis, Leptospira, seroprevalencia, ganado bovino

Abstract: A cross-sectional study was performed in the Huetar Norte region of Costa Rica (20102012) to establish seroprevalence to Leptospira spp among dairy cows. This region presents a high incidence of human infection. It also has the largest milk production index in the country. A total of 2375 individual samples was processed using the microscopic agglutination test (MAT), which corresponded to 36 farms belonging to the Dairy Farmers' Cooperative Cooperativa de Productores de Leche Dos Pinos. Samples yielding a titer`1:160 were considered positive. Under

Autor de correspondencia: jsequeira@inciensa.sa.cr 


\begin{abstract}
that criterion a $9.13 \%$, overall seroprevalence was obtained but titers as high as 1:5210 were observed in clinically healthy animals. Seroprevalence among farms was heterogeneous ranging from 0-25\%. The most frequently detected serovars were Pomona (15.7\%); Tarassovi (16.6\%), and Hebdomadis (25.8\%). A statistically significant association was detected $(p<0.05)$ between certain serovars and farms. Similarly, water sources used by the farms showed a statistical association $(p<0.05)$ with seroprevalence to Leptospira spp. $(p>0.05)$. This association was not identified for the animal's age or breed ( $p>0.05)$.
\end{abstract}

Keywords: Leptospirosis, Leptospira, seroprevalence, dairy cattle

\title{
Introducción
}

La leptospirosis humana es la enfermedad zoonótica con mayor incidencia en el mundo (Cachay \& Vinetz 2005); los datos sugieren que Costa Rica no es la excepción.

En este sentido, sobresale la provincia de Limón, en su totalidad; Turrialba en Cartago; Aguirre y los cantones del Sur de Puntarenas, así como Los Chiles y San Carlos en Alajuela. Datos del Ministerio de Salud, para el 2008, reportan una tasa de notificación de poco más de 10 por cada 100000; reconociendo, además, que esta no refleja la realidad, debido al marcado subregistro y subdiagnóstico de la misma (Ministerio de Salud de Costa Rica 2009).

Este hecho, junto con la compleja epidemiología del agente, que incluye múltiples reservorios animales, hace necesaria la ejecución de investigaciones que permitan identificar las principales serovariedades circulantes en el país, así como la frecuencia con que estas pueden estar infectando los animales domésticos con los cuales se convive diariamente.

En el ganado bovino, la leptospirosis es responsable, sobre todo, de pérdidas en la reproducción. De esta manera, puede presentar natimuertos, abortos y/o nacimiento de animales débiles e infertilidad, aunque de manera menos frecuente (Andicoberry et al. 2001).

Debido ala compleja etiología de los trastornos reproductivosen bovinos; ala amplia presencia de títulos de anticuerpos para Leptospira spp en bovinos de Costa Rica (Muñoz 1999), el diagnóstico definitivo de la enfermedad, en nuestro país, es más una excepción. Otras manifestaciones, en bovinos, incluyen la presencia de agalactia transitoria pero sin tumefacción de la glándula mamaria, lo cual, repercute en pérdidas económicas. Por último, en animales jóvenes, se reporta, aunque con poca frecuencia, un cuadro agudo grave que incluye fiebre, ictericia, hemorragias y hemoglobinuria, situación que frecuentemente es de curso fatal (Muñoz 1999).

En Costa Rica son limitados, en algunos casos inconsistentes, los reportes del impacto y prevalencia de esta infección en el bovino (Muñoz 1999); lo cual dificulta cuantificar la verdadera prevalencia de anticuerpos contra Leptospira spp. en esta especie, con variaciones que van de $86.9 \%$ positividad, a un $64.5 \%$ el año siguiente (Quirós 1995; Muñoz 1999). Tales variaciones, con la metodología empleada, es difícil explicarlas. No se han realizado análisis similares en los años siguientes.

Este evento tiene un importante impacto en la salud humana; destacan zonas bien identificadas con circulación del agente (Ministerio de Salud de Costa Rica 2009). Debido 
a este comportamiento y su subregistro, se hace necesaria la ejecución de investigaciones que nos permitan conocer la verdadera presentación de este evento en el país. Este trabajo pretende aportar información sobre la seroprevalencia del agente en ganado bovino lechero, con el fin de dar bases sólidas para instaurar medidas de control de la enfermedad más adecuadas y efectivas.

\section{Materiales y métodos}

El estudio se desarrolla como un estudio transversal para determinar la seroprevalencia de anticuerpos anti Leptospira spp. en las fincas lecheras asociadas de la cooperativa Dos Pinos. La colecta de muestras se realizó en el periodo 2010 y 2012.

El universo de la investigación se estableció con el ganado bovino de las fincas lecheras de la región Huetar Norte, las cuales vendían su leche a la empresa Dos Pinos, al momento del muestreo. Se consideró, como unidades de análisis, el ganado bovino de las fincas de socios de la cooperativa.

Se incluyó, en este estudio, a todo animal bovino mayor de 18 meses. No se realizó distingo de sexo o raza. Se excluyó los animales que recibieron vacunas contra cualquier serovariedad de Leptospira spp. en los 18 meses anteriores al muestreo, y, ninguno tenía manifestaciones clínicas de enfermedad.

Se estableció, para el muestreo, un total de 2.500 animales, por razones de conveniencia y complejidad diagnóstica. La afijación se hizo mediante la metodología de conglomerados, en una sola etapa, en la cual, cada finca se consideró como unidades primarias de muestreo (UPM), las cuales se seleccionaron al azar, mediante tablas de números aleatorios. De las fincas elegidas, se muestreó todas las unidades secundarias de muestreo (USM), mediante la recolección de muestras sanguíneas de todo el ganado vacuno de la finca, siempre y cuando cumplieran los criterios de inclusión; además, que los criterios de exclusión permitieran su estadía en el grupo muestreado.

Con el fin de analizar la información básica de los animales muestreados y de la finca, se analizó las variables correspondientes a la identificación de la finca; ubicación y coordenadas geográficas de la finca; edad aproximada, raza y sexo del animal; tipos de fuentes de agua, así como los resultados obtenidos, por el laboratorio, según título de anticuerpos y serogrupo identificado en este ensayo.

Con el fin de identificar la presencia de anticuerpos contra Leptospira spp, a toda muestra se realizó una prueba de tamizaje mediante la técnica de microaglutinación (MAT) por microscopía de campo oscuro, en el cual cada una se evaluó contra 16 serovariedades de Leptospira spp, correspondientes, cada una, a un serogrupo diferente (Cuadro 1). 
Cuadro 1. Serovariedades y serogrupos utilizados en la técnica de MAT, región Huetar Norte, Costa Rica, 2010-2012

\begin{tabular}{cc}
\hline Serovaridad & Serogrupo que representa \\
\hline Arenal & Javanica \\
Australis & Australis \\
Autumnalis & Autumnalis \\
Batavie & Batavie \\
Canicola & Canicola \\
Castellonis & Ballum \\
Copenhageni & Icterohaemorrhagiae \\
Cynopteri & Cynopteri \\
Grippotyphosa & Grippotyphosa \\
Hardjo & Sejroe \\
Hebdomadis & Hebdomadis \\
Javanica & Javanica \\
Panama & Panama \\
Pomona & Pomona \\
Pyrogenes & Pomona \\
Tarassovi & Tarassovi \\
\hline
\end{tabular}

Estas serovariedades son las recomendadas, por la Organización Mundial de la Salud, para este diagnóstico (ILS/WHO 2003); además de la serovariedad Arenal, la cual fue aislada en esta región de estudio y es considerada como una cepa autóctona (Valverde et al. 2008). Debido a que la especificidad de esta técnica es baja para la detección específica de la serovariedad infectante, dado el reconocimiento antigénico cruzado en ésta técnica serológica, se incidieron los resultados a nivel de serogrupo, probablemente infectante.

Las muestras se analizaron en el laboratorio del Centro Nacional de Referencia de Leptospirosis (CNRL) del INCIENSA, en diluciones de 1:30 y 1:300, con el fin de contrarrestar cualquier efecto zonal. Las muestras positivas, en cualquiera de estos títulos, fueron tituladas contra la serovariedad correspondiente, mediante diluciones dobles a partir de diluciones 1:20 hasta un título de 1:20480. El título final se consideró como aquel en el cual se observó una aglutinación del $50 \%$ de las bacterias.

Títulos iguales o menores de 1:80 se consideraron como negativos. Este estudio consideró, como títulos positivos por Leptospira spp., aquellos que, en el análisis serológico, mostraron un título de anticuerpos a uno o más de los antígenos leptospirales detectados por la prueba de microaglutinación (MAT) mayor o igual a 1:160 (Dastis-Bendala et al. 1999). 
Cuadro 2. Resultado de serología por leptospirosis, según número de finca, región Huetar Norte, Costa Rica, 2010-2012.

\begin{tabular}{|c|c|c|c|c|c|}
\hline \multirow{3}{*}{$\begin{array}{l}\text { Número } \\
\text { Finca }\end{array}$} & \multicolumn{4}{|c|}{ Resultado Serología } & \multirow{3}{*}{ Total } \\
\hline & \multicolumn{2}{|c|}{ Negativo } & \multicolumn{2}{|c|}{ Positivo } & \\
\hline & $\mathbf{n}$ & $\%$ & $\mathbf{n}$ & $\%$ & \\
\hline 1 & 116 & 77,3 & 34 & 22,7 & 150 \\
\hline 2 & 61 & 95,3 & 3 & 4,7 & 64 \\
\hline 3 & 24 & 75,0 & 8 & 25,0 & 32 \\
\hline 4 & 106 & 80,3 & 26 & 19,7 & 132 \\
\hline 5 & 48 & 96,0 & 2 & 4,0 & 50 \\
\hline 6 & 30 & 100,0 & 0 & 0,0 & 30 \\
\hline 7 & 62 & 76,5 & 19 & 23,5 & 81 \\
\hline 8 & 86 & 87,8 & 12 & 12,2 & 98 \\
\hline 9 & 48 & 87,3 & 7 & 12,7 & 55 \\
\hline 10 & 123 & 91,1 & 12 & 8,9 & 135 \\
\hline 11 & 39 & 100,0 & 0 & 0,0 & 39 \\
\hline 12 & 84 & 95,5 & 4 & 4,5 & 88 \\
\hline 13 & 38 & 92,7 & 3 & 7,3 & 41 \\
\hline 14 & 50 & 96,2 & 2 & 3,8 & 52 \\
\hline 15 & 23 & 100,0 & 0 & 0,0 & 23 \\
\hline 16 & 103 & 93,6 & 7 & 6,4 & 110 \\
\hline 17 & 32 & 97,0 & 1 & 3,0 & 33 \\
\hline 18 & 118 & 95,9 & 5 & 4,1 & 123 \\
\hline 19 & 131 & 93,6 & 9 & 6,4 & 140 \\
\hline 20 & 53 & 100,0 & 0 & 0,0 & 53 \\
\hline 21 & 33 & 100,0 & 0 & 0,0 & 33 \\
\hline 22 & 45 & 97,8 & 1 & 2,2 & 46 \\
\hline 23 & 50 & 96,2 & 2 & 3,8 & 52 \\
\hline 24 & 115 & 94,3 & 7 & 5,7 & 122 \\
\hline 25 & 53 & 93,0 & 4 & 7,0 & 57 \\
\hline 26 & 29 & 93,5 & 2 & 6,5 & 31 \\
\hline 27 & 36 & 100,0 & 0 & 0,0 & 36 \\
\hline 28 & 36 & 90,0 & 4 & 10,0 & 40 \\
\hline 29 & 28 & 93,3 & 2 & 6,7 & 30 \\
\hline 30 & 60 & 82,2 & 13 & 17,8 & 73 \\
\hline 31 & 105 & 89,7 & 12 & 10,3 & 117 \\
\hline 32 & 30 & 85,7 & 5 & 14,3 & 35 \\
\hline 33 & 24 & 100,0 & 0 & 0,0 & 24 \\
\hline 34 & 56 & 91,8 & 5 & 8,2 & 61 \\
\hline 35 & 60 & 93,8 & 4 & 6,3 & 64 \\
\hline 36 & 23 & 92,0 & 2 & 8,0 & 25 \\
\hline Total & 2158 & 90,9 & 217 & $9,1 \%$ & 2375 \\
\hline
\end{tabular}


El análisis de datos se realizó utilizando los programas Epiinfo 3.5.4 (Dean AG et al. 2011) y SPSS 17.0 (SPSS Inc. Chicago, Illinois, EE. UU.). Se realizó un análisis descriptivo de la información considerando la distribución espacial de las fincas y las características del ganado muestreado.

\section{Resultados}

Se recolectó y analizó 2.375 muestras, (95\% del total estimado), provenientes de 36 fincas; 2.279 fueron hembras (96.0\%).

En el proceso de tamizaje previo de las muestras, se obtuvo un resultado positivo en 1.172 muestras, al menos a una serovariedad de Leptospira spp (49.3\%). De las muestras positivas en el tamizaje, 217 fueron positivas a títulos de al menos en 1:160 al momento de titularlas, para una prevalencia general de $9.14 \%$ (IC95\% 8.02-10.38). Sin embargo, este porcentaje varía entre las diferentes fincas de manera significativa $\left(X^{2}=136.9 ; p=0,000\right)$; como se puede observar en el Cuadro 2 en el cual se observa fincas con prevalencias de hasta un $25 \%$.

El Cuadro 3 contiene la distribución de la edad aproximada de los animales muestreados. Se pudo observar asociación estadística entre la edad de los animales y los resultados obtenidos en el análisis serológico por la técnica de MAT $\left(X^{2}=13.6 ; p=0.001\right.$, prueba exacta Fisher). Se observó mayor positividad a más edad del animal.

Entre los grupos analizados se observa una diversa conformación en cuanto a razas. Como se observa en el Cuadro 4, la mayoría de los animales pertenecía a la raza Holstein, Jersey o varios cruces. No se observó asociación entre las características raciales del animal y los resultados obtenidos por MAT ( $\left.X^{2}=14.3 ; p=0,357\right)$. Un total de 32 animales resultó sin dato para raza.

No se observó asociación significativa entre el sexo del animal y el resultado por MAT $\left(X^{2}=0.057 ; p=0.499\right)$.

De las 217 muestras positivas, se observó que 125 (57.6\%) fueron con títulos de 1:160; $49(22.6 \%)$ con títulos de 1:320; 26 (12.0\%) a títulos de 1:640; 14 (6.5\%) con títulos de 1:1280; 1 muestra (0.5\%) fue positiva a $1: 2560 \mathrm{y}$, finalmente, 2 muestras fueron con título de $1: 5120$ (0.9\%).

En la Figura 1 se aprecia la localización geográfica de las fincas muestreadas y analizadas, con resultado positivo por anticuerpos anti-Leptospira spp en la zona Huetar Norte de Costa Rica. 
Cuadro 3. Distribución de resultados por MAT obtenidos según edad aproximada, región Huetar Norte, Costa Rica, 2010-2012.

\begin{tabular}{lrrrrrr}
\hline \multirow{2}{*}{ Edad } & \multicolumn{3}{c}{ Resultado Serología } & \multicolumn{2}{c}{ Total } \\
\cline { 2 - 6 } & \multicolumn{2}{c}{ Negativo } & \multicolumn{2}{c}{ Positivo } & & \\
\cline { 2 - 7 } & $\mathbf{n}$ & $\mathbf{\%}$ & $\mathbf{n}$ & $\mathbf{\%}$ & $\mathbf{n}$ & $\mathbf{\%}$ \\
\hline Ternera (o) (hasta los 18 meses) & 63 & 3,0 & 17 & 7,8 & 80 & 3,5 \\
\hline Novilla (o) (de 18 meses a 3 años) & 1703 & 15,3 & 40 & 18,4 & 358 & 15,6 \\
\hline Vaca Adulta (más de 3 años) & 2084 & 100,0 & 217 & 100,0 & 2301 & 100,0 \\
\hline Total & & & & & & \\
\hline
\end{tabular}

Cuadro 4. Distribución de frecuencias de razas analizadas, región Huetar Norte, Costa Rica, 2010-2012.

\begin{tabular}{lcc}
\hline Razas & Frecuencia & Porcentaje \\
\hline Angus & 1 & 0,0 \\
Ayshire & 1 & 0,0 \\
Holstein-Simmental & 1 & 0,0 \\
Brahman & 2 & 0,1 \\
Gyr & 5 & 0,2 \\
Brahman-Jersey & 15 & 0,6 \\
Guernsey & 29 & 1,2 \\
Holstein-Brahman & 38 & 1,6 \\
Pardo & 38 & 1,6 \\
Otras Razas & 55 & 2,3 \\
Otros Cruces & 249 & 10,6 \\
Chumeca (Holstein-Jersey) & 535 & 22,8 \\
Jersey & 668 & 28,5 \\
Holstein & 706 & 30,1 \\
\hline Total & 2343 & 100,0 \\
\hline
\end{tabular}




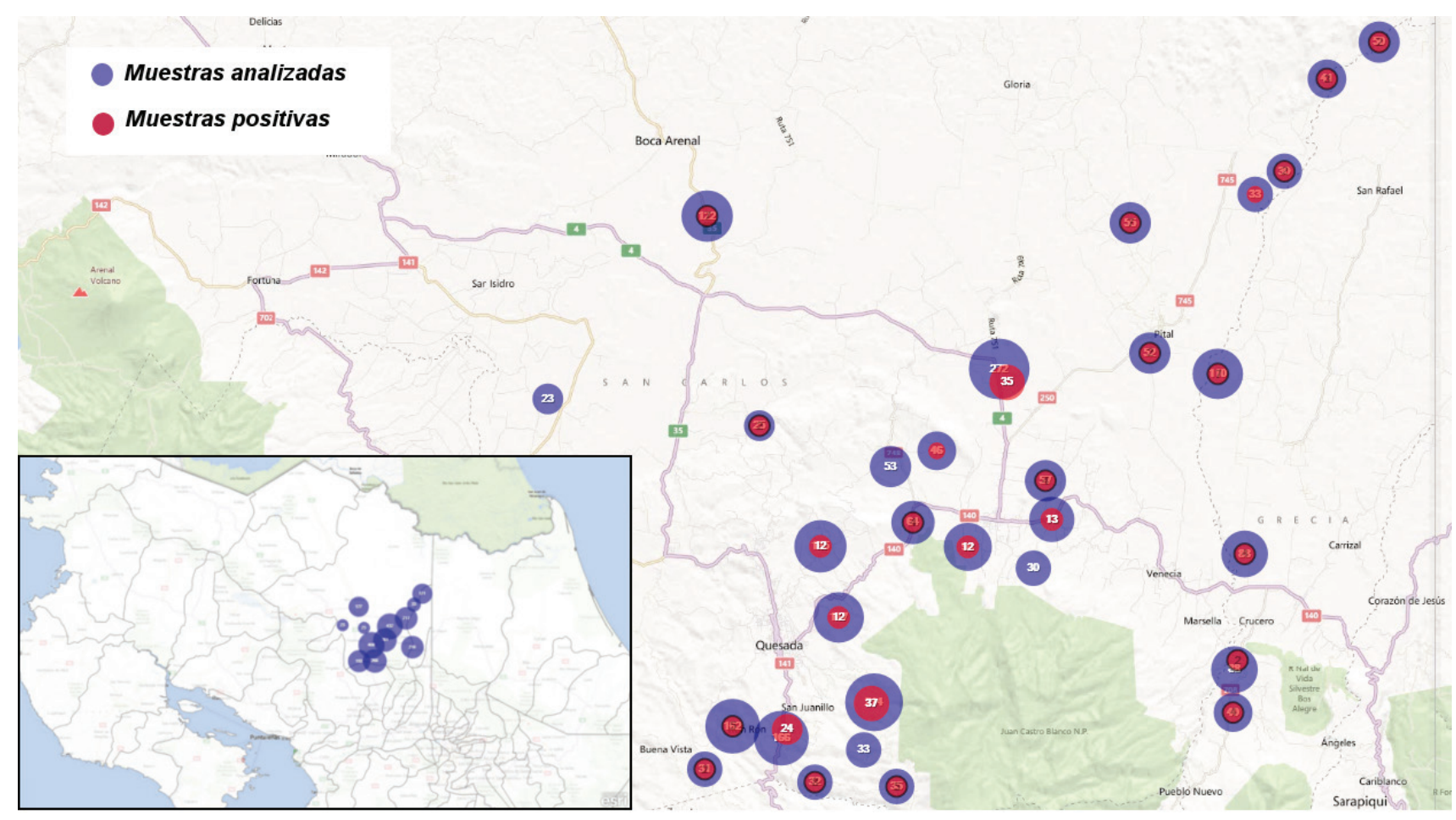

Figura 1. Mapa de las muestras recolectadas y analizadas, región Huetar Norte, Costa Rica, 2010-2012.

Como se observa, en el Cuadro 5, las muestras positivas en la titulación se presentaron, en mayor porcentaje, contra las serovariedades Hebdomadis, Tarassovi y Pomona, de la batería diagnóstica utilizada en el estudio. La Figura 2 muestra la distribución de los serogrupos y serovariedades con mayor frecuencia de positividad.

Al analizar el comportamiento de los títulos de anticuerpos, contra los distintos serogrupos analizados, se observa que Canicola y Tarassovi son los serogrupos que logran presentar títulos iguales o superiores a 1:2560 (Cuadro 6). 
Cuadro 5. Distribución de frecuencias de serogrupos y serovariedades en muestras positivas por MAT, región Huetar Norte, Costa Rica, 2010-2012.

\begin{tabular}{llcc}
\hline Serogrupo & Serovariedad & Frecuencia & Porcentaje \\
\hline Grippotyphosa & Grippotyphosa & 1 & $0,5 \%$ \\
Cynopteri & Cynopteri & 2 & $0,9 \%$ \\
Autumnalis & Autumnalis & 4 & $1,8 \%$ \\
Pyrogenes & Pyrogenes & 4 & $1,8 \%$ \\
Ballum & Castellonis & 5 & $2,3 \%$ \\
Varias* & Varias* & 16 & $7,4 \%$ \\
Sejroe & Hardjo & 17 & $7,8 \%$ \\
Icterohaemorrhagiae & Copenhageni & 20 & $9,2 \%$ \\
Canicola & Canicola & 22 & $10,1 \%$ \\
Pomona & Pomona & 34 & $15,7 \%$ \\
Tarassovi & Tarassovi & 36 & $16,6 \%$ \\
Hebdomadis & Hebdomadis & 56 & $25,8 \%$ \\
\hline
\end{tabular}

*Resultado positivo a más de una serovariedad con el mismo título de anticuerpos.

Cuadro 6. Distribución de los títulos de anticuerpos según serogrupo en muestras positivas por MAT, región Huetar Norte, Costa Rica, 2010-2012.

\begin{tabular}{|c|c|c|c|c|c|c|c|}
\hline \multirow{2}{*}{ Serogrupo } & \multicolumn{6}{|c|}{ Título de anticuerpos } & \multirow{2}{*}{ Total } \\
\hline & $1 / 160$ & $1 / 320$ & $1 / 640$ & $1 / 1280$ & $1 / 2560$ & $1 / 5120$ & \\
\hline Grippotyphosa & 1 & 0 & 0 & 0 & 0 & 0 & 1 \\
\hline Cynopteri & 2 & 0 & 0 & 0 & 0 & 0 & 2 \\
\hline Autumnalis & 3 & 1 & 0 & 0 & 0 & 0 & 4 \\
\hline Pyrogenes & 1 & 2 & 0 & 1 & 0 & 0 & 4 \\
\hline Ballum & 5 & 0 & 0 & 0 & 0 & 0 & 5 \\
\hline Varias & 7 & 6 & 2 & 1 & 0 & 0 & 16 \\
\hline Sejroe & 8 & 2 & 6 & 1 & 0 & 0 & 17 \\
\hline Icterohaemorrhagiae & 15 & 4 & 0 & 1 & 0 & 0 & 20 \\
\hline Canicola & 12 & 4 & 3 & 1 & 1 & 1 & 22 \\
\hline Pomona & 22 & 7 & 4 & 1 & 0 & 0 & 34 \\
\hline Tarassovi & 24 & 7 & 2 & 2 & 0 & 1 & 36 \\
\hline Hebdomadis & 25 & 16 & 9 & 6 & 0 & 0 & 56 \\
\hline Total & 125 & 49 & 26 & 14 & 1 & 2 & 217 \\
\hline
\end{tabular}


Como se aprecia en la Figura 4, algunos serogrupos se caracterizan por distribuirse de manera heterogénea entre las fincas analizadas $\left(X^{2}=457.39 ; p=0,000\right)$. Ejemplo de esto es la distribución de los serogrupos Ballum, Cynopteri, Grippotyphosa y Pyrogenes, los cuales se identificaron en pocas fincas. En tanto que los serogrupos con mayor frecuencia (Hebdomadis, Tarassovi y Pomona) se observan en gran cantidad de fincas lecheras de la muestra seleccionada. En el Cuadro 7, se observa este comportamiento con mayor detalle.

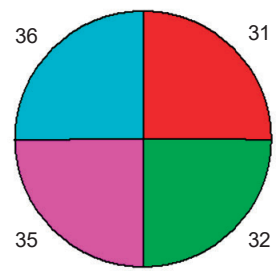

Autumnalis

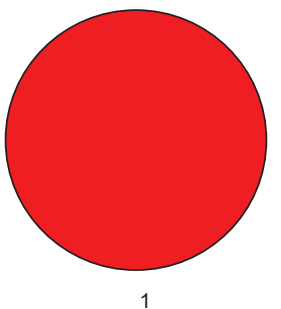

Grippotyphosa

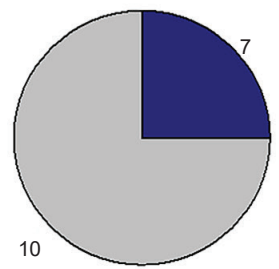

Pyrogenes

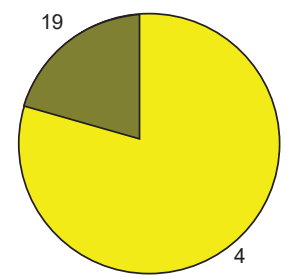

Ballum

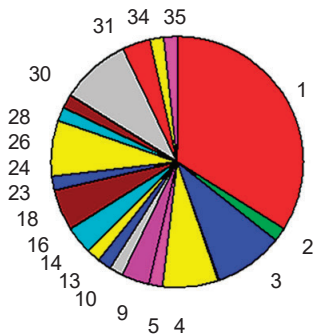

Hebdomadis

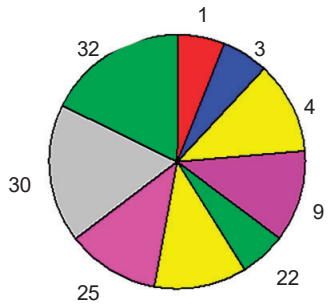

24

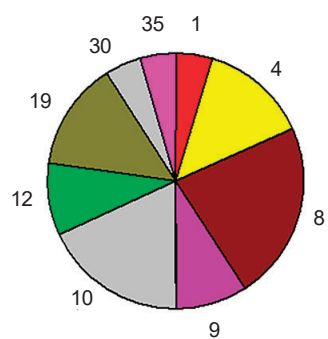

Canicola

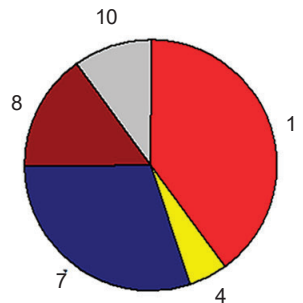

Icterohaemorrhagiae

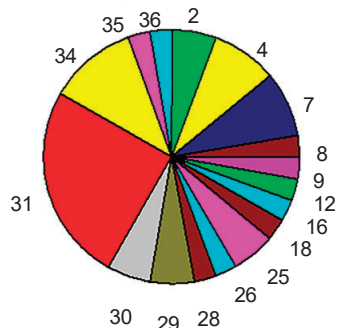

Tarassovi

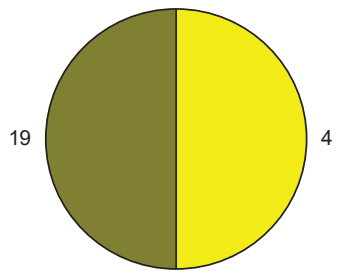

Cynopteri

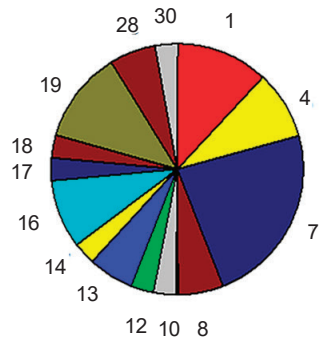

Pomona

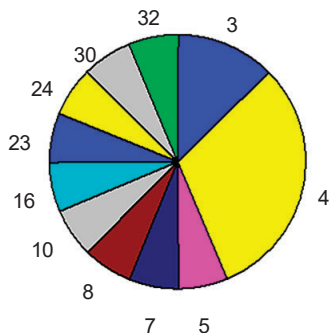

Varios Serogrupos

Figura 2. Distribución de serogrupos según número de finca, región Huetar Norte, Costa Rica, 2010-2012 
Rev. Ciencias Veterinarias, Vol. 34, № 1, [23-37], ISSN: 2215-4507, enero-junio, 2016 DOI: http://dx.doi.org/10.15359/rcv.34-1.2

URL: http://www.revistas.una.ac.cr/index.php/veterinaria/index

Cuadro 7. Frecuencias de serogrupos por serología según número de finca, región Huetar Norte, Costa Rica, 2010-2012.

\begin{tabular}{|c|c|c|c|c|c|c|c|c|c|c|c|c|c|c|c|c|c|c|c|c|c|c|c|c|c|c|}
\hline \multicolumn{27}{|c|}{ Serogrupo } \\
\hline \multirow[t]{2}{*}{$\begin{array}{l}\text { Número } \\
\text { de Finca }\end{array}$} & \multicolumn{2}{|r|}{ 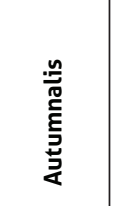 } & \multicolumn{2}{|r|}{ 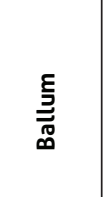 } & \multicolumn{2}{|r|}{ 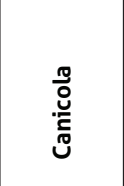 } & \multicolumn{2}{|r|}{ 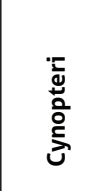 } & \multicolumn{2}{|r|}{ 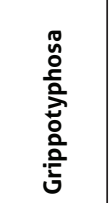 } & \multicolumn{2}{|c|}{ 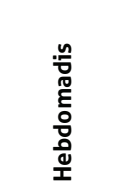 } & \multicolumn{2}{|r|}{ 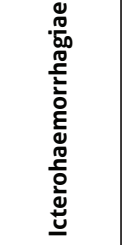 } & \multicolumn{2}{|r|}{ 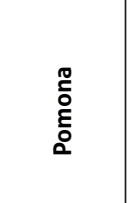 } & \multicolumn{2}{|r|}{ 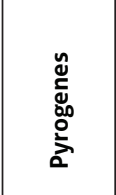 } & \multicolumn{2}{|r|}{ ڤั. } & \multicolumn{2}{|r|}{ 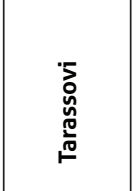 } & \multicolumn{2}{|c|}{ 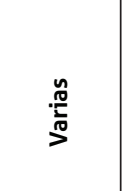 } & \multicolumn{2}{|r|}{ 芯 } \\
\hline & $n$ & $\%$ & $n$ & $\%$ & $n$ & $\%$ & $n$ & $\%$ & $n$ & $\%$ & $n$ & $\%$ & $n$ & $\%$ & $n$ & $\%$ & $n$ & $\%$ & $n$ & $\%$ & $\bar{n}$ & $\%$ & $n$ & $\%$ & $\mathrm{n}$ & $\%$ \\
\hline 1 & 0 & 0 & 0 & 0 & 1 & 4,5 & 0 & 0 & 1 & 100 & 19 & 33,9 & 8 & 40,0 & 4 & 11,8 & 0 & 0 & 1 & 5,9 & 0 & 0 & 0 & 0 & 34 & 15,7 \\
\hline 2 & 0 & 0 & 0 & 0 & 0 & 0 & 0 & 0 & 0 & 0 & 1 & 1,8 & 0 & 0 & 0 & 0 & 0 & 0 & 0 & 0 & 2 & 5,6 & 0 & 0 & 3 & 1,4 \\
\hline 3 & 0 & 0 & 0 & 0 & 0 & 0 & 0 & 0 & 0 & 0 & 5 & 8,9 & 0 & 0 & 0 & 0 & 0 & 0 & 1 & 5,9 & 0 & 0 & 2 & 12,5 & 8 & 3,7 \\
\hline 4 & 0 & 0 & 4 & 80,0 & 3 & 13,6 & 1 & 50,0 & 0 & 0 & 4 & 7,1 & 1 & 5,0 & 3 & 8,8 & 0 & 0 & 2 & 11,8 & 3 & 8,3 & 5 & 31,3 & 26 & 12 \\
\hline 5 & 0 & 0 & 0 & 0 & 0 & 0 & 0 & 0 & 0 & 0 & 1 & 1,8 & 0 & 0 & 0 & 0 & 0 & 0 & 0 & 0 & 0 & 0 & 1 & 6,3 & 2 & 0,9 \\
\hline 7 & 0 & 0 & 0 & 0 & 0 & 0 & 0 & 0 & 0 & 0 & 0 & 0 & 6 & 30,0 & 8 & 23,5 & 1 & 25,0 & 0 & 0 & 3 & 8,3 & 1 & 6,3 & 19 & 8,8 \\
\hline 8 & 0 & 0 & 0 & 0 & 5 & 22,7 & 0 & 0 & 0 & 0 & 0 & 0 & 3 & 15,0 & 2 & 5,9 & 0 & 0 & 0 & 0 & 1 & 2,8 & 1 & 6,3 & 12 & 5,5 \\
\hline 9 & 0 & 0 & 0 & 0 & 2 & 9,1 & 0 & 0 & 0 & 0 & 2 & 3,6 & 0 & 0 & 0 & 0 & 0 & 0 & 2 & 11,8 & 1 & 2,8 & 0 & 0 & 7 & 3,2 \\
\hline 10 & 0 & 0 & 0 & 0 & 4 & 18,2 & 0 & 0 & 0 & 0 & 1 & 1,8 & 2 & 10,0 & 1 & 2,9 & 3 & 75,0 & 0 & 0 & 0 & 0 & 1 & 6,3 & 12 & 5,5 \\
\hline 12 & 0 & 0 & 0 & 0 & 2 & 9,1 & 0 & 0 & 0 & 0 & 0 & 0 & 0 & 0 & 1 & 2,9 & 0 & 0 & 0 & 0 & 1 & 2,8 & 0 & 0 & 4 & 1,8 \\
\hline 13 & 0 & 0 & 0 & 0 & 0 & 0 & 0 & 0 & 0 & 0 & 1 & 1,8 & 0 & 0 & 2 & 5,9 & 0 & 0 & 0 & 0 & 0 & 0 & 0 & 0 & 3 & 1,4 \\
\hline 14 & 0 & 0 & 0 & 0 & 0 & 0 & 0 & 0 & 0 & 0 & 1 & 1,8 & 0 & 0 & 1 & 2,9 & 0 & 0 & 0 & 0 & 0 & 0 & 0 & 0 & 2 & 0,9 \\
\hline 16 & 0 & 0 & 0 & 0 & 0 & 0 & 0 & 0 & 0 & 0 & 2 & 3,6 & 0 & 0 & 3 & 8,8 & 0 & 0 & 0 & 0 & 1 & 2,8 & 1 & 6,3 & 7 & 3,2 \\
\hline 17 & 0 & 0 & 0 & 0 & 0 & 0 & 0 & 0 & 0 & 0 & 0 & 0 & 0 & 0 & 1 & 2,9 & 0 & 0 & 0 & 0 & 0 & 0 & 0 & 0 & 1 & 0,5 \\
\hline 18 & 0 & 0 & 0 & 0 & 0 & 0 & 0 & 0 & 0 & 0 & 3 & 5,4 & 0 & 0 & 1 & 2,9 & 0 & 0 & 0 & 0 & 1 & 2,8 & 0 & 0 & 5 & 2,3 \\
\hline 19 & 0 & 0 & 1 & 20,0 & 3 & 13,6 & 1 & 50,0 & 0 & 0 & 0 & 0 & 0 & 0 & 4 & 11,8 & 0 & 0 & 0 & 0 & 0 & 0 & 0 & 0 & 9 & 4,1 \\
\hline 22 & 0 & 0 & 0 & 0 & 0 & 0 & 0 & 0 & 0 & 0 & 0 & 0 & 0 & 0 & 0 & 0 & 0 & 0 & 1 & 5,9 & 0 & 0 & 0 & 0 & 1 & 0,5 \\
\hline 23 & 0 & 0 & 0 & 0 & 0 & 0 & 0 & 0 & 0 & 0 & 1 & 1,8 & 0 & 0 & 0 & 0 & 0 & 0 & 0 & 0 & 0 & 0 & 1 & 6,3 & 2 & 0,9 \\
\hline 24 & 0 & 0 & 0 & 0 & 0 & 0 & 0 & 0 & 0 & 0 & 4 & 7,1 & 0 & 0 & 0 & 0 & 0 & 0 & 2 & 11,8 & 0 & 0 & 1 & 6,3 & 7 & 3,2 \\
\hline 25 & 0 & 0 & 0 & 0 & 0 & 0 & 0 & 0 & 0 & 0 & 0 & 0 & 0 & 0 & 0 & 0 & 0 & 0 & 2 & 11,8 & 2 & 5,6 & 0 & 0 & 4 & 1,8 \\
\hline 26 & 0 & 0 & 0 & 0 & 0 & 0 & 0 & 0 & 0 & 0 & 1 & 1,8 & 0 & 0 & 0 & 0 & 0 & 0 & 0 & 0 & 1 & 2,8 & 0 & 0 & 2 & 0,9 \\
\hline 28 & 0 & 0 & 0 & 0 & 0 & 0 & 0 & 0 & 0 & 0 & 1 & 1,8 & 0 & 0 & 2 & 5,9 & 0 & 0 & 0 & 0 & 1 & 2,8 & 0 & 0 & 4 & 1,8 \\
\hline 29 & 0 & 0 & 0 & 0 & 0 & 0 & 0 & 0 & 0 & 0 & 0 & 0 & 0 & 0 & 0 & 0 & 0 & 0 & 0 & 0 & 2 & 5,6 & 0 & 0 & 2 & 0,9 \\
\hline 30 & 0 & 0 & 0 & 0 & 1 & 4,5 & 0 & 0 & 0 & 0 & 5 & 8,9 & 0 & 0 & 1 & 2,9 & 0 & 0 & 3 & 17,6 & 2 & 5,6 & 1 & 6,3 & 13 & 6 \\
\hline 31 & 1 & \begin{tabular}{|l}
25,0 \\
\end{tabular} & 0 & 0 & 0 & 0 & 0 & 0 & 0 & 0 & 2 & 3,6 & 0 & 0 & 0 & 0 & 0 & 0 & 0 & 0 & 9 & 25 & 0 & 0 & 12 & 5,5 \\
\hline 32 & 1 & 25,0 & 0 & 0 & 0 & 0 & 0 & 0 & 0 & 0 & 0 & 0 & 0 & 0 & 0 & 0 & 0 & 0 & 3 & 17,6 & 0 & 0 & 1 & 6,3 & 5 & 2,3 \\
\hline 34 & 0 & 0 & 0 & 0 & 0 & 0 & 0 & 0 & 0 & 0 & 1 & 1,8 & 0 & 0 & 0 & 0 & 0 & 0 & 0 & 0 & 4 & 11,1 & 0 & 0 & 5 & 2,3 \\
\hline 35 & 1 & 25,0 & 0 & 0 & 1 & 4,5 & 0 & 0 & 0 & 0 & 1 & 1,8 & 0 & 0 & 0 & 0 & 0 & 0 & 0 & 0 & 1 & 2,8 & 0 & 0 & 4 & 1,8 \\
\hline 36 & 1 & 25,0 & 0 & 0 & 0 & 0 & 0 & 0 & 0 & 0 & 0 & 0 & 0 & 0 & 0 & 0 & 0 & 0 & 0 & 0 & 1 & 2,8 & 0 & 0 & 2 & 0,9 \\
\hline Total & 4 & 100,0 & 5 & 100,0 & 22 & 100,0 & 2 & 100,0 & 1 & 100,0 & 56 & 100,0 & 20 & 100,0 & 34 & 100,0 & 4 & 100,0 & 17 & 100,0 & 36 & 100,0 & 16 & 100,0 & 217 & 100,0 \\
\hline
\end{tabular}


En cuanto a las distintas fuentes de agua, aproximadamente el $47 \%$ de los animales recibe agua de cañería; en menor proporción de río o nacientes. Se observó asociación entre las fuentes de agua de las fincas y los resultados en el análisis serológico por la técnica de MAT $\left(X^{2}=39.7\right.$; $p=0.000$, prueba exacta Fisher). Estas fuentes y su frecuencia se observan en el Cuadro 8.

Cuadro 8. Distribución de los resultados por MAT según fuente de agua utilizada, región Huetar Norte, Costa Rica, 2010-2012.

\begin{tabular}{lrrrrrrr}
\hline \multirow{2}{*}{ Fuentes de Agua } & \multicolumn{3}{c}{ Resultado Serología } & \multicolumn{2}{c}{ Total } \\
\cline { 2 - 6 } & \multicolumn{2}{c}{ Negativo } & \multicolumn{2}{c}{ Positivo } & & \\
\cline { 2 - 6 } & $\mathbf{n}$ & $\%$ & $\mathbf{n}$ & $\%$ & $\mathbf{n}$ & $\%$ \\
Pozo & 30 & 1,4 & 5 & 2,3 & 35 & 1,5 \\
Cañería Municipal & 530 & 24,8 & 25 & 11,5 & 555 & 23,6 \\
Río o Naciente & 694 & 32,5 & 55 & 25,3 & 749 & 31,8 \\
Mixta & 881 & 41,3 & 132 & 60,8 & 1013 & 43,1 \\
Total & 2135 & 100,0 & 217 & 100,0 & 2352 & 100,0 \\
\hline
\end{tabular}

\section{Discusión}

Las muestras analizadas para el diagnóstico de la leptospirosis en la Región Huetar Norte en ganado vacuno, de acuerdo con el estudio, muestran que la seroprevalencia de este evento, aunque importante, no es tan alto como, en ocasiones anteriores, se ha reportado (Quirós 1995; Muñoz 1999). Sin embargo, es importante mencionar las diferencias en el nivel de seropositividad entre fincas. Se pudo identificar fincas sin anticuerpos detectables anti-Leptospira spp; otras con un $25 \%$ de seropositividad.

Esta variación podría estar relacionada con diferencias en la ecología y manejo entre fincas. Otro hallazgo es la distribución heterogénea de los serogrupos en algunas fincas. En este sentido, la variación de condiciones ecológicas entre fincas, también puede estar jugando un papel significativo; además, la colecta de muestras se realizó a lo largo de casi 2 años, tanto en la estación seca como lluviosa, lo cual puede incidir en estas variaciones (Leanne et al. 2011). Ambos hallazgos deben aclararse en posteriores estudios pues, en este, el objetivo es el perfil serológico del agente en la región.

Importante hacer notar que los serogrupos más significativos, en cuanto a frecuencia en el diagnóstico por MAT (Hebdomadis, Tarassovi y Pomona), se identificaron en gran cantidad de fincas, por lo cual, los esfuerzos para el control de la infección en la zona deben considerar estos serogrupos, para garantizar un mayor impacto. La detección de Hebdomadis y Tarassovi 
representa un hecho interesante, dado que estudios anteriores, en la región (Maranda 2003; González, Maranda \& Romero 2007), no los incluyeron. Adicionalmente, el serogrupo Sejroe (al cual pertenece la serovariedad Hadjo) y considerado en Norte América como el más importante en bovinos (Prescott et al. 1988), fue menos frecuente en la región Huetar Norte.

Maranda, 2003; en su estudio evaluó una de las vacunas disponibles en Costa Rica contra Leptospira spp. Esta vacuna se elabora con cepas pertenecientes a diferentes serovariedades y serogrupos, que frecuentemente causan infección en Norte América: Hardjo (serogrupo Sejroe); Pomona, (serogrupo Pomona); Grippotyphosa, (serogrupo Grippotyphosa); Icterohaemorragiae, (serogrupo Icterohaemorrhagiae) y Canicola (serogrupo Canicola), sin embargo, no pudo detectar protección.

En el estudio, a excepción del serogrupo Pomona, las representadas, en las vacunas citadas, no están ( $\leq 10 \%)$ entre las de mayor prevalencia en las fincas muestreadas; lo cual podría explicar los resultados de Maranda (2003).

Si bien el diagnóstico clínico de la leptospirosis se confirma mediante muestras pareadas, con el fin de establecer seroconversión, el propósito de la investigación es establecer la presencia de anticuerpos específicos en las fincas analizadas; por tanto, exposición al agente en animales clínicamente sanos. Por tal razón, solo se analizó muestras únicas de cada individuo. La frecuencia y severidad, con que las serovariedades detectadas inducen procesos clínicos en la región, debe analizarse en estudios posteriores.

La información serológica obtenida ofrece una idea de las serovariedades circulantes; incluso la posibilidad de realizar recomendaciones generales para su prevención, la cual puede ser de utilidad al productor.

Está fuera del alcance del trabajo hacer comentarios sobre la peligrosidad de las serovariedades detectadas. Sin embargo, la literatura indica que, se debe realizar el aislamiento pero, además, considerar el tamaño del inóculo infectante; la condición del huésped como accidental o de mantenimiento del agente; edad y el estatus inmune del paciente, entre otros (Faine 1999; Levett 2001).

La relevancia de las secreciones animales, la contaminación de fuentes de agua y alimentos en la transmisión del agente es ampliamente reconocida (Adler \& de la Peña Moctezuma 2010). Por tal razón, durante el estudio, se colectó información sobre las fuentes de agua utilizadas con los animales.

Un porcentaje significativo de fincas con animales seropositivos (40.6\%) utilizaba agua proveniente de cañerías, exposición que no se observa asociado con este evento. Sin embargo, es importante mencionar que las fincas, adicional a la cañería, también poseen quebradas y riachuelos como fuentes de agua durante varios meses del año, lo cual dificulta la interpretación de los resultados.

Se observó una asociación significativa entre la edad aproximada del animal y los resultados por serología, siendo la vaca adulta de más de 3 años la que cursa con una positividad alta para Leptospira spp. lo cual puede ser el resultado de una exposición más prolongada al agente. 
La técnica del MAT, a pesar de ser el método de referencia de este evento, es limitada en su interpretación y alcance, debido a la reactividad cruzada que puede ocurrir entre las cepas pertenecientes al mismo serogrupo y entre serogrupos. Esta técnica serológica no permite identificar, correctamente, la serovariedad y cepa causante de la infección. Únicamente, mediante el aislamiento es posible identificar al agente causante de la infección (Chirathaworn et al. 2014). Por tal razón, es recomendable, para futuros estudios, realizar el aislamiento e identificación de cepas de Leptospira spp. e intentar asociar esto con la serología, clínica y epidemiología observada.

La incorporación de cepas autóctonas adicionales en las baterías diagnósticas utilizadas en el país, incrementaría la sensibilidad del MAT en la detección de esta patología. Estos aislamientos, una vez caracterizados, serían potenciales candidatos para la elaboración de vacunas específicas a la circulación de cepas en las zonas con circulación del agente, lo cual permitirá una mejor prevención de la enfermedad.

\section{Referencias}

Adler B., de la Peña Moctezuma A. 2010. Leptospira and Leptospirosis. Vet. Microbiol. 140 (3-4): 287-296. doi: 10.1016/j.vetmic.2009.03.012

Andicoberry, A., C.García Peña, F. \& Ortega Mora, L. 2001. Epidemiología, diagnóstico y control de la leptospirosis bovina. Investigación Agraria. Producción y Sanidad Animales 16(2): 205-226.

Cachay, E. R. \& Vinetz, J. M. 2005. A global research agenda for leptospirosis. Journal of Postgraduade Medicine 51(3): 174-178.

Chirathaworn, C., Inwattana, R., Poovorawan, Y. \& Suwancharoen, D. 2014. Interpretation of microscopic agglutination test for leptospirosis diagnosis and seroprevalence. Asian Pacific Journal of Tropical Biomedicine, 4(Suppl 1), S162-S164. http://doi. org/10.12980/APJTB.4.2014C580

Dastis-Bendala, C., Villar-Conde, E., Marin-Leone, I., Manzanares-Torne, L., Perez-Lozano, M. J., Cano-Fuentes, G. \& Pumarola-Suñe, T. 1996. Prospective serological study of leptospirosis in Southern Spain. European Journal of Epidemiology, 12(3), 257-262.

Dean AG, Arner TG, Sunki GG, F. R., Lantinga M, Sangam S, Zubieta JC, Sullivan KM, Brendel KA, G. Z. \& Fontaine N, Shu M, Fuller G, Smith DC, Nitschke DA, and F. R. 2011. Epi Info ${ }^{T M}$, a database and statistics program for public health professionals. CDC.

Faine S. 1999. Leptospira and Leptospirosis. Second Edition. Medisci Press. Melbourne Australia.

González, R., Maranda, L. \& Romero, J. J. 2007. Efecto de la vacunación anti-Leptospira en bovinos lecheros sobre la producción de anticuerpos anti-Leptospira en trabajadores de lecherías de cuatro zonas de Costa Rica. Revista Costarricense de Salud Pública 16(30): 39-45. 
Levett P.N. 2001.Leptospirosis.Clin. Microbiol.14:296-236.

ILS/WHO. 2003. Human leptospirosis: guidance for diagnosis, surveillance and control. Malta, p. 114.

Maranda, L. 2003. Estudio Experimental Sobre Efectos de la Vacuna Leptofern-5 en Ganado Lechero, en Costa Rica, 2001. Universidad de Davis, California.

Ministerio de Salud de Costa Rica. 2005. Memoria Institucional 2004. San José, Costa Rica. Ministerio de Salud de Costa Rica. 2009. Memoria Institucional 2008. San José, Costa Rica.

Muñoz, M. 1999. Leptospirosis: Revisión bibliográfica y análisis de la enfermedad en Costa Rica. Universidad Nacional.

Prescott J.F. 1988. Seroprevalence and association with abortion en cattle in Ontario.Can. Vet. J. 52:210-213

Quirós, L. 1995. Leptospirosis: Seroepidemiología en bovinos de Costa Rica y la situación epidemiológica de bovinos y cerdos de la Región Huetar Norte. Universidad Nacional.

Universidad de Costa Rica. 2014. Centro Centroamericano de Población. http://censos.ccp. ucr.ac.cr. (Accessed January 13, 2014)

Van De Weyer LM, Hendrick S, Rosengren L, Waldner CL. 2011. An update on leptospirosis in beef herds from western Canada: Serum antibody titers and vaccination practices. Can Vet J. 52:619-626.

Valverde, M., Ramírez, J., Montes de Oca, L., Goris, M., Ahmed, N. \& Hartskeerl, R. 2008. Arenal, a new Leptospira serovar of serogroup Javanica, isolated from a patient in Costa Rica. Infection, Genetics and Evolution 8(5): 529-33. doi:10.1016/j.meegid.2008.02.008 